\title{
Mandala-inspired representation of the turbulent energy cascade
}

\author{
Maxime Bassenne, ${ }^{*}$ Hyunji Jane Bae, and Adrián Lozano-Durán \\ Center for Turbulence Research, Stanford University, Stanford, California 94305-3024, USA
}

(Received 27 January 2018; published 17 October 2018)

\begin{abstract}
This paper is associated with a poster winner of a 2017 APS/DFD Milton van Dyke Award for work presented at the DFD Gallery of Fluid Motion. The original poster is available from the Gallery of Fluid Motion, https://doi.org/10.1103/APS.DFD.2017.GFM.P0026
\end{abstract}

DOI: 10.1103/PhysRevFluids.3.100505

Understanding the cascading process of turbulent kinetic energy from large-scale fluid motions to small-scale and lesser-scale fluid motions is critical to modeling strategies for geophysical and industrial flows. The phenomenological explanation of the transfer of energy across scales was introduced in the classical paper by Kolmogorov [1], but the concept of a turbulent cascade in terms of interactions among eddies was proposed earlier by Richardson [2] and later by Obukhov [3]. Since then, many detailed investigations, mostly in isotropic turbulence, have greatly advanced our understanding of high-Reynolds-number turbulent flows.

Attempts to unravel the mechanisms behind the cascade have relied on varying but complementary physical rationales. A classic explanation is given in terms of vortex stretching acting across scales $[4,5]$. Other approaches have directly tested the original idea of Richardson [2] in terms of eddy breakdown [6,7] or multifractal models for the statistical properties of the energy transfer [8]. Notwithstanding the efforts, the cascading process remains one of the most challenging problems in turbulence due to its multiscale nature. The advent of wavelet methods has opened new avenues for analyzing nonlinear processes in turbulence $[9,10]$, partly due to their ability to unfold signals into both space and scale, simultaneously.

In this work, the continuous wavelet transform is applied to one-dimensional instantaneous velocity signals obtained from direct numerical simulation of three-dimensional isotropic turbulence. Details about the data can be found in [7,11]. The algorithm uses the complex-valued Morlet wavelet [12], converting one-dimensional physical-space velocity signals into two-dimensional arrays of wavelet coefficients that represent the local velocity fluctuations at a given scale around a fixed position. The complex wavelet coefficients, of which only the real parts are visualized in the present analysis, are subsequently mapped into polar coordinates. The radial and azimuthal coordinates represent inverse scale and position, respectively.

Figure 1 shows the resulting polar plots. They are visual evidence of the Richardson turbulent energy cascade. In particular, the pitchfork pattern that describes the distribution of energy across scales around a fixed position (moving from the center to the edge at a fixed azimuthal angle) reveals its fractal character [13]. The effect of the Reynolds number is highlighted in Figs. 1(a)-1(d). The energy-containing eddies represented in the core of the figures at small-radius values exhibit little sensitivity to the increase in Reynolds number. On the contrary, the inertial-range scales shown

\footnotetext{
*Corresponding author: bassenne@ stanford.edu
}

Published by the American Physical Society under the terms of the Creative Commons Attribution 4.0 International license. Further distribution of this work must maintain attribution to the author(s) and the published article's title, journal citation, and DOI. 


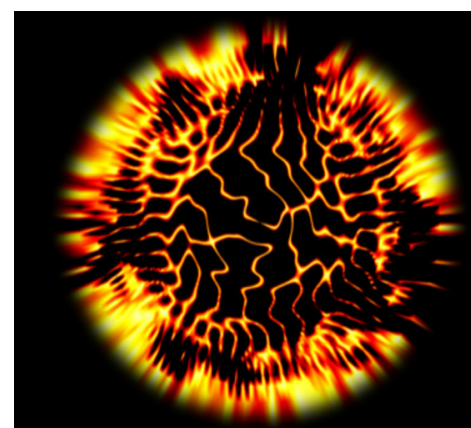

(a)

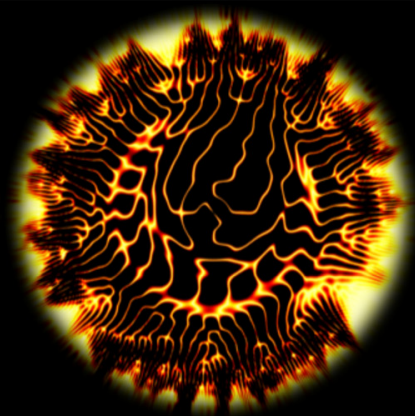

(b)

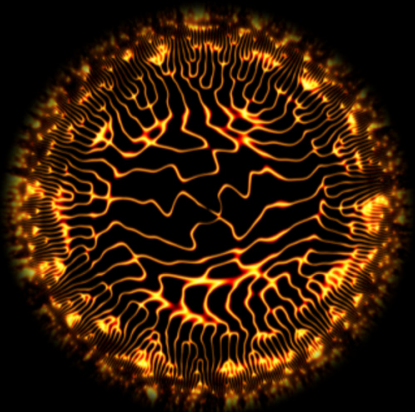

(c)

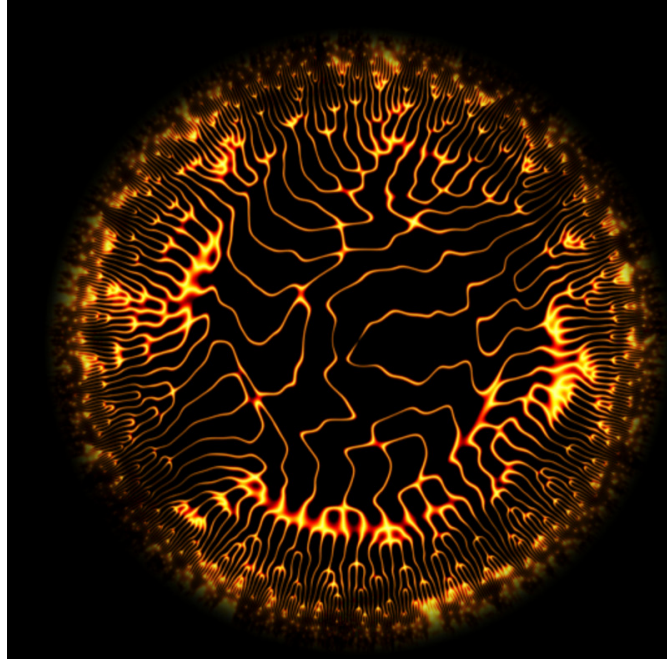

(d) (c)

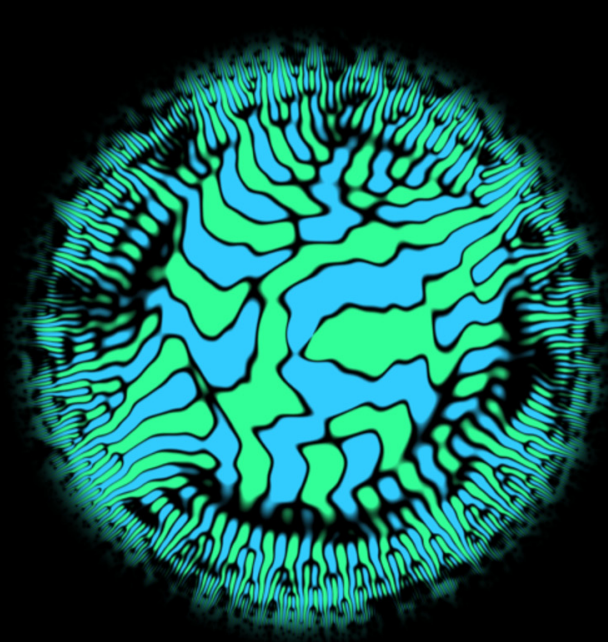

(e)

FIG. 1. Polar plots of the wavelet transform of one-dimensional velocity signals extracted from threedimensional isotropic turbulence data at different Taylor-microscale Reynolds number: (a) $\operatorname{Re}_{\lambda}=140$, (b) $\operatorname{Re}_{\lambda}=380$, (c) $\operatorname{Re}_{\lambda}=1100$, and (d) and (e) $\operatorname{Re}_{\lambda}=2300$. In each panel, wavelet coefficients are normalized by the scale-dependent extremum. In (a)-(d) colors (orange) highlight zero wavelet coefficients. In (e) colors represent positive (blue) and negative (green) wavelet coefficients, descriptive of turbulent structures.

at larger-radius values contain an increasing number of cascading stages, visually demonstrating the larger-scale separation between the energy-containing and the dissipation ranges. The turbulent structures are shown for the largest Reynolds number in Fig. 1(e), which is a complementary version of Fig. 1(d).

The authors are grateful to Prof. Yukio Kaneda and Prof. Takashi Ishihara for providing the data and thank Prof. Geoffrey Spedding and Mark Fischer for useful discussions. M.B. acknowledges support from the Advanced Simulation and Computing program of the US Department of Energy's National Nuclear Security Administration via the PSAAP-II Center at Stanford (Grant No. 107908). H.J.B. and A.L.-D. acknowledge the support of NASA Transformative Aeronautics Concepts Program (Grant No. UNIX15AU93A). 
[1] A. N. Kolmogorov, The local structure of turbulence in incompressible viscous fluid for very large Reynolds numbers, Dokl. Akad. Nauk SSSR 30, 301 (1941).

[2] L. F. Richardson, Weather Prediction by Numerical Process (Cambridge University Press, Cambridge, 1922).

[3] A. M. Obukhov, On the distribution of energy in the spectrum of turbulent flow, Izv. Akad. Nauk USSR, Ser. Geogr. Geofiz. 5, 453 (1941).

[4] A. Lozano-Durán, M. Holzner, and J. Jiménez, Multiscale analysis of the topological invariants in the logarithmic region of turbulent channels at a friction Reynolds number of 932, J. Fluid Mech. 803, 356 (2016).

[5] S. Goto, Y. Saito, and G. Kawahara, Hierarchy of antiparallel vortex tubes in spatially periodic turbulence at high Reynolds numbers, Phys. Rev. Fluids 2, 064603 (2017).

[6] A. Lozano-Durán and J. Jiménez, Time-resolved evolution of coherent structures in turbulent channels: characterization of eddies and cascades, J. Fluid Mech. 759, 432 (2014).

[7] J. I. Cardesa, A. Vela-Martín, and J. Jiménez, The turbulent cascade in five dimensions, Science 357, 782 (2017).

[8] U. Frisch and R. J. Donnelly, Turbulence: The Legacy of A. N. Kolmogorov (AIP, New York, 1996).

[9] M. Farge, Wavelet transforms and their applications to turbulence, Annu. Rev. Fluid Mech. 24, 395 (1992).

[10] K. Schneider and O. Vasilyev, Wavelet methods in computational fluid dynamics, Annu. Rev. Fluid Mech. 42, 473 (2010).

[11] T. Ishihara, K. Morishita, M. Yokokawa, A. Uno, and Y. Kaneda, Energy spectrum in high-resolution direct numerical simulations of turbulence, Phys. Rev. Fluids 1, 082403 (2016).

[12] A. Grossmann and J. Morlet, Decomposition of hardy functions into square integrable wavelets of constant shape, SIAM J. Math. Anal. 15, 723 (1984).

[13] F. Argoul, A. Arneodo, G. Grasseau, Y. Gagne, E. J. Hopfinger, and U. Frisch, Wavelet analysis of turbulence reveals the multifractal nature of the richardson cascade, Nature 338, 51 (1989). 